\title{
Quantum Counterpart of Classical Equipartition of Energy
}

\author{
Jerzy Łuczka ${ }^{1,2}$ \\ Published online: 16 May 2020 \\ (c) The Author(s) 2020
}

\begin{abstract}
It is shown that the recently proposed quantum analogue of classical energy equipartition theorem for two paradigmatic, exactly solved models (i.e., a free Brownian particle and a dissipative harmonic oscillator) also holds true for all quantum systems which are composed of an arbitrary number of non-interacting or interacting particles, subjected to any confining potentials and coupled to thermostat with arbitrary coupling strength.
\end{abstract}

Keywords Quantum systems · Equipartition of energy · Quantum analogue

\section{Introduction}

In classical statistical physics, the theorem on equipartition of kinetic energy is one of the most universal relation [1,2]. It states that for a system in thermodynamic equilibrium of temperature $T$, the mean kinetic energy $E_{k}$ per one degree of freedom is equal to $E_{k}=k_{B} T / 2$, where $k_{B}$ is the Boltzmann constant $[3,4]$. It does not depend on a number of particles in the system, the form of the potential force which acts on them, the form of interaction between particles and strength of coupling between the system and thermostat. It depends only on the thermostat temperature $T$. On the contrary, for quantum systems, the mean kinetic energy is not equally shared among all degrees of freedom and the theorem fails. The quite natural question arises whether one can formulate a similar and universal relation for the mean kinetic energy of quantum systems at the thermodynamic equilibrium state. Recently, in a series of papers [5-7], the authors have proposed quantum analogue of the classical energy equipartition theorem. For a system of one degree of freedom this quantum counterpart, which is called the energy partition theorem, has the following appealing form:

$$
E_{k}=\int_{0}^{\infty} \mathcal{E}_{k}(\omega) \mathbb{P}(\omega) d \omega,
$$

Communicated by Abishek Dhar.

Jerzy Łuczka

jerzy.luczka@us.edu.pl

1 Institute of Physics, University of Silesia, 41-500 Chorzow, Poland

2 Institute of Physics, University of Augsburg, 86135 Augsburg, Germany 
where

$$
\mathcal{E}_{k}(\omega)=\frac{\hbar \omega}{4} \operatorname{coth}\left[\frac{\hbar \omega}{2 k_{B} T}\right]
$$

has the same form as thermally averaged kinetic energy of the harmonic oscillator with the frequency $\omega$ weakly coupled to thermostat of temperature $T$ [8]. The function $\mathbb{P}(\omega)$ has all properties of a probability density on a positive half-line of real numbers meaning that

$$
\begin{aligned}
& \mathbb{P}(\omega) \geq 0, \\
& \int_{0}^{\infty} \mathbb{P}(\omega) d \omega=1 .
\end{aligned}
$$

The explicit form of $\mathbb{P}(\omega)$ has been derived for two exactly solved quantum systems: a free Brownian particle [5] and a dissipative harmonic oscillator [6]. In these papers [5,6], thermostat is composed of quantum harmonic oscillators (à la Caldeira-Leggett [9-11]) and the above interpretation of $\mathcal{E}_{k}(\omega)$ as their mean kinetic energy per one degree of freedom is fully justified. Because $\mathbb{P}(\omega)$ is a probability density, Eq. (1) can be rewritten in the form

$$
E_{k}=\left\langle\mathcal{E}_{k}\right\rangle
$$

where $\left\langle\mathcal{E}_{k}\right\rangle$ is a mean value of the function $\mathcal{E}_{k}(\xi)$ of some random variable $\xi$ distributed according to the probability density $\mathbb{P}$. In the Caldeira-Leggett model, $\xi$ can be interpreted as a random frequency of harmonic oscillators forming the thermostat which should be infinitely extended, i.e., the thermodynamic limit for the thermostat should be carried out in order to guarantee a continuous spectrum of the thermostat oscillators frequencies.

\section{Universal Relation for Kinetic Energy of Quantum Systems}

Here, we want to prove a relation similar to Eq. (1) for a class of quantum systems for which the concept of kinetic energy has sense (e.g spin systems are outside of this class). More precisely, we study a quantum system $S$ coupled to a heat bath (thermostat, environment) $B$. The composite system $S+B$ is in a Gibbs equilibrium state of temperature $T$ defined by the density operator

$$
\rho=Z^{-1} e^{-H / k_{B} T}, \quad Z=\operatorname{Tr}\left[e^{-H / k_{B} T}\right]
$$

and

$$
H=H_{S}+H_{\text {int }}+H_{B}
$$

is the Hamiltonian of the composite system $S+B$. Next,

$$
H_{S}=\sum_{j} \frac{p_{j}^{2}}{2 M_{j}}+\sum_{j} U_{S}\left(x_{j}\right)+\sum_{j, k} V_{S}\left(x_{j}, x_{k}\right)
$$

is the Hamiltonian of the system $S$ and

$$
H_{i n t}=\sum_{j, n} \lambda_{j n} V\left(x_{j}, X_{n}\right)
$$

is the Hamiltonian of interaction of the system $S$ with the thermostat $B$. Finally, $H_{B}$ is the Hamiltonian of thermostat $B$. Its explicit form is now not relevant. The set of parameters 
$\left\{\lambda_{j n}\right\}$ characterizes the coupling strength. The coordinate and momentum operators $\left\{x_{j}, p_{j}\right\}$ refer to the system $S$ and the operators $\left\{X_{n}\right\}$ refer to the thermostat $B$. All coordinate and momentum operators obey canonical equal-time commutation relations. We assume that all components of the Hamiltonian (7) fulfil required conditions to ensure a well defined thermodynamic equilibrium state of the composite system $S+B$ in the thermodynamic limit for the thermostat.

Theorem 1 The mean kinetic energy per one degree of freedom of the system $S$ can be expressed in a universal form as

$$
E_{k}^{(j)}=\left\langle\mathcal{E}_{k}\right\rangle^{(j)}=\int_{0}^{\infty} \mathcal{E}_{k}(\omega) \mathbb{P}_{j}(\omega) d \omega,
$$

where

$$
E_{k}^{(j)}=\left\langle\frac{p_{j}^{2}}{2 M_{j}}\right\rangle=\operatorname{Tr}\left[\frac{p_{j}^{2}}{2 M_{j}} \rho\right]
$$

and $\mathcal{E}_{k}(\omega)$ is given by Eq. (2). The function $\mathbb{P}_{j}(\omega)$ is a probability density which obeys conditions (3) and (4).

The explicit form of the probability density $\mathbb{P}_{j}(\omega)$ is presented below.

Proof of Theorem 1 To prove the relation (10), we apply the fluctuation-dissipation relation of the Callen-Welton type $[12,13]$. One can exploit the results derived e.g. in the LandauLifshitz book [14] [see Eq. (124.10)] or in the Zubarev book [15] [see Eq. (17.19g)]. We apply them to the momentum operator $p_{j}$ of the system $S$. Without loss of generality we assume that the average momentum $\left\langle p_{j}\right\rangle=0$ at the equilibrium state and then one obtains

$$
\left\langle p_{j}^{2}\right\rangle=\frac{\hbar}{\pi} \int_{0}^{\infty} \operatorname{coth}\left[\frac{\hbar \omega}{2 k_{B} T}\right] \chi_{j j}^{\prime \prime}(\omega) d \omega,
$$

where $\chi_{j j}^{\prime \prime}(\omega)$ is the imaginary part of the generalized susceptibility,

$$
\chi_{j j}(\omega)=\chi_{j j}^{\prime}(\omega)+i \chi_{j j}^{\prime \prime}(\omega) .
$$

The real part is an even function and the imaginary part is an odd function,

$$
\chi_{j j}^{\prime}(\omega)=\chi_{j j}^{\prime}(-\omega), \quad \chi_{j j}^{\prime \prime}(\omega)=-\chi_{j j}^{\prime \prime}(-\omega) .
$$

The generalized susceptibility $\chi_{j j}(\omega)$ is the Fourier transform

$$
\chi_{j j}(\omega)=\int_{-\infty}^{\infty} \mathrm{e}^{i \omega t} G_{j j}(t) d t
$$

of the response function $G_{j j}(t)$ which in fact is the retarded thermodynamic Green function [15], namely,

$$
G_{j j}(t)=\frac{i}{\hbar} \theta(t)\left\langle\left[p_{j}(t), p_{j}(0)\right]\right\rangle,
$$

where $\theta(t)$ is the Heaviside step function and

$$
p_{j}(t)=\exp (i H t / \hbar) p_{j}(0) \exp (-i H t / \hbar)
$$

is the Heisenberg representation of the momentum $p_{j}(0)$. The averaging in Eq. (16) is over the Gibbs canonical statistical operator (6). 
Now, we compare Eqs. (10) and (12), and obtain the expression for $\mathbb{P}_{j}(\omega)$ in the form

$$
\mathbb{P}_{j}(\omega)=\frac{2}{\pi M_{j}} \frac{\chi_{j j}^{\prime \prime}(\omega)}{\omega} .
$$

Hence, we obtain the formal expression for $\mathbb{P}_{j}(\omega)$. However, we have to show that it can be interpreted as a probability density.

Corollary The function $\mathbb{P}_{j}(\omega)$ assumes non-negative values for all positive values of the argument $\omega$.

Proof We use the spectral representation of $\chi_{j j}^{\prime \prime}(\omega)$ in the form (see e.g. the equation just above Eq. (124.9) in the Landau-Lifshitz book [14]),

$$
\chi_{j j}^{\prime \prime}(\omega)=\frac{\pi}{\hbar}\left(1-\mathrm{e}^{-\hbar \omega / k_{B} T}\right) \sum_{m, n} \rho_{n}\left|p_{n m}\right|^{2} \delta\left(\hbar \omega+E_{n}-E_{m}\right),
$$

where $p_{n m}$ are the matrix elements of the momentum operator in the basis of the eigenstates of the total Hamiltonian $H, E_{n}$ are the eigenvalues of $H$ and the population factor is $\rho_{n}=$ $Z^{-1} \exp \left(-E_{n} / k_{B} T\right)$. From the form of this relation we see that for all positive $\omega$ the function $\chi_{j j}^{\prime \prime}(\omega)$ is positive and not zero. In fact, it is a well-known that $\chi_{j j}^{\prime \prime}(\omega)$ is positive and is also named an absorptive part of the susceptibility $\chi_{j j}(\omega)$, see also the text below Eq. (123.11) in [14]. Hence, also $\mathbb{P}_{j}(\omega)$ given by Eq. (18) is positive for all positive values of $\omega$.

Theorem 2 The function $\mathbb{P}_{j}(\omega)$ defined by Eq. (18) is normalized to unity,

$$
\int_{0}^{\infty} \mathbb{P}_{j}(\omega) d \omega=\frac{1}{M_{j}} \frac{2}{\pi} \int_{0}^{\infty} \frac{\chi_{j j}^{\prime \prime}(\omega)}{\omega} d \omega=1 .
$$

Proof of Theorem 2 According to the Kramers-Kronig dispersion relation

$$
\chi_{j j}^{\prime}(\omega)=\frac{2}{\pi} \mathcal{P} \int_{0}^{\infty} \frac{u \chi_{j j}^{\prime \prime}(u)}{u^{2}-\omega^{2}} d u,
$$

where $\mathcal{P}$ denotes the principal value of the integral. Its value at $\omega=0$ reads

$$
\chi_{j j}(0)=\frac{2}{\pi} \mathcal{P} \int_{0}^{\infty} \frac{\chi_{j j}^{\prime \prime}(u)}{u} d u,
$$

where we utilize the relation $\chi_{j j}^{\prime}(0)=\chi_{j j}(0)$ which follows from (13) and (14) for $\omega=0$. The rhs of this equation is related to Eq. (20). Alternatively, one can apply Eq. (123.19) in the Landau-Lifshitz book [14] which reads

$$
\chi_{j j}(i \omega)=\frac{2}{\pi} \int_{0}^{\infty} \frac{u \chi_{j j}^{\prime \prime}(u)}{\omega^{2}+u^{2}} d u
$$

and for $\omega=0$ it takes the same value as (22). On the other hand, from Eqs. (15) and (16) it follows that

$$
\chi_{j j}(0)=\int_{-\infty}^{\infty} G_{j j}(t) d t=\frac{i}{\hbar} \int_{0}^{\infty}\left\langle\left[p_{j}(t), p_{j}(0)\right]\right\rangle d t
$$


We observe that the problem of normalization of $\mathbb{P}_{j}(\omega)$ in Eq. (18) is converted to the problem whether the equality

$$
\chi_{j j}(0)=M_{j}
$$

holds true for the Hamiltonian (7)-(9).

This may seem surprising at first glance since $\chi_{j j}(0)$ does not depend on the form of the potential, interaction, temperature and parameters of the Hamiltonian, but it depends only on mass $M_{j}$ of the particle considered.

In the next step, we prove that the relation (25) indeed holds true for a general form of the Hamiltonian (7). We start from the Heisenberg equations of motion for coordinate operators of the system $S$, namely,

$$
\frac{d x_{j}(t)}{d t}=\frac{i}{\hbar}\left[H, x_{j}(t)\right]=\frac{p_{j}(t)}{M_{j}} .
$$

We insert it into Eq. (24) and obtain

$$
\begin{aligned}
\chi_{j j}(0)= & \frac{i M_{j}}{\hbar} \lim _{\epsilon \rightarrow 0^{+}} \int_{0}^{\infty} \mathrm{e}^{-\epsilon t} \frac{d}{d t}\left\langle\left[x_{j}(t), p_{j}(0)\right]\right\rangle d t \\
= & \left.\frac{i M_{j}}{\hbar} \mathrm{e}^{-\epsilon t}\left\langle\left[x_{j}(t), p_{j}(0)\right]\right\rangle\right|_{0} ^{\infty} \\
& +\frac{i M_{j}}{\hbar} \lim _{\epsilon \rightarrow 0^{+}} \epsilon \int_{0}^{\infty} \mathrm{e}^{-\epsilon t}\left\langle\left[x_{j}(t), p_{j}(0)\right]\right\rangle d t,
\end{aligned}
$$

where we use a well-known limiting procedure with the $\epsilon$-term to ensure convergence of the integral [16]. The integral in the last line is finite and therefore this term tends to zero as $\epsilon \rightarrow 0$. In the first term, for the upper limit $t \rightarrow \infty$ the expression tends to zero. For the lower limit, $\left\langle\left[x_{j}(0), p_{j}(0)\right]\right\rangle=i \hbar$. Thus it finishes proofs of the relation (25) and normalization of the function $\mathbb{P}_{j}(\omega)$ defined by Eq. (18).

\section{Comments and Discussion}

1. The formula (10) is a generalization of the classical energy equipartition theorem. It fulfils elementary conditions for generalization: Indeed, in the high temperature limit

$$
\operatorname{coth}\left[\frac{\hbar \omega}{2 k_{B} T}\right] \approx \frac{2 k_{B} T}{\hbar \omega}, \quad \mathcal{E}_{k}(\omega) \approx k_{B} T / 2
$$

and Eq. (10) reduces to its classical counterpart

$$
E_{k}^{(j)}=\frac{1}{2} k_{B} T \int_{0}^{\infty} \mathbb{P}_{j}(\omega) d \omega=\frac{1}{2} k_{B} T
$$

because of normalization of $\mathbb{P}_{j}(\omega)$. We want to notice that Callen and Welton in their 'historical' paper [12] missed the normalization: see Eq. (4.11) therein.

2. It has to be stressed that the formula (10) is universal, however, the mean kinetic energy $E_{k}^{(j)}$ depends not only on temperature of the system (as in the classical case) but also, via the probability density $\mathbb{P}_{j}(\omega)$, on a number of particles in the system, the form of the potential which acts on them, the form of interaction between particles and strength of coupling between the system and thermostat. 
3. If $H$ is the Hamiltonian of the composite system $S+B$ then all regimes, from weak to strong coupling with thermostat, can be analyzed. However, if $H=H_{S}$ (there is no explicit interaction with thermostat $B$ ) then it means that only the weak coupling limit can be considered because averaging is over the Gibbs canonical density operator $\rho_{S} \propto \exp \left(-H_{S} / k_{B} T\right)$ valid in the weak coupling limit.

4. There are no specific assumptions regarding thermostat $B$ : It should be infinitely extended and satisfying the Kubo-Martin-Schwinger conditions expressing periodicity of Green's functions in imaginary time [16,17].

5. The factor $\mathcal{E}_{k}(\omega)$ in Eq. (10) is the same as mean kinetic energy of a quantum harmonic oscillator in the Gibbs state $\rho_{O} \propto \exp \left(-H_{O} / k_{B} T\right)$, where $H_{O}$ is the Hamiltonian of the harmonic oscillator [8],

$$
\mathcal{E}_{k}(\omega)=\frac{1}{2 m}\left\langle p^{2}\right\rangle=\frac{\hbar \omega}{4} \operatorname{coth} \frac{\hbar \omega}{2 k_{B} T} .
$$

It depends on the frequency $\omega$ of the harmonic oscillator but not upon its mass $m$. However, in the considered model (7)-(9), a harmonic oscillator does not occur at all. It is a consequence of the above point 4 and the linear response theory [18].

6. As an example, we demonstrate how the above theory works for a free Brownian particle coupled to thermostat which is a collection of harmonic oscillators [5]. What we need is the explicit form of the momentum operator $p(t)$ which has been calculated e.g. in Ref. [5], see Eq. (7) therein. It reads

$$
\begin{aligned}
p(t)= & R(t) p(0)-\int_{0}^{t} R(t-u) \gamma(u) d u x(0) \\
& +\int_{0}^{t} R(t-u) \eta(u) d u
\end{aligned}
$$

where $R(t)$ and $\gamma(t)$ are the response function and the memory kernel of the generalized Langevin equation. The operator $\eta(t)$ models quantum thermal noise and is expressed by thermostat operators which commute with the system operators. In Eq. (16), only the second term in r.h.s. of Eq. (31) contributes to the commutator yielding the Green function

$$
G(t)=\theta(t) \int_{0}^{t} R(t-u) \gamma(u) d u .
$$

The susceptibility $\chi(\omega)$ is a Fourier transform of the Green function $G(t)$ which is a convolution in (32) of two scalar functions $R(t)$ and $\gamma(t)$. Therefore as a result we obtain

$$
\chi(\omega)=\hat{R}_{L}(-i \omega) \hat{\gamma}_{L}(-i \omega),
$$

i.e., it is expressed by a product of two Laplace transforms $\hat{R}_{L}(z)$ and $\hat{\gamma}_{L}(z)$ of the functions $R(t)$ and $\gamma(t)$, respectively. For the free Brownian particle of mass $M$ the Laplace transforms of $R(t)$ reads [5]

$$
\hat{R}_{L}(z)=\frac{M}{M z+\hat{\gamma}_{L}(z)}
$$

and the generalized susceptibility takes the form

$$
\chi(\omega)=\frac{M \hat{\gamma}_{L}(-i \omega)}{-i \omega M+\hat{\gamma}_{L}(-i \omega)} .
$$

It is seen that for any form of the memory function $\gamma(t)$ the value of susceptibility at zero frequency is the particle mass, $\chi(0)=M$. 
In conclusion, applying the fluctuation-dissipation relation we demonstrate that Eq. (10) is valid for arbitrary quantum systems described by the Hamiltonian (7)-(9) and being at the thermodynamic equilibrium state. The probability distribution is of the form (18), where the susceptibility $\chi_{j j}(\omega)$ is the Fourier transform of the retarded thermodynamic Green function (16). The formula (10) can be called the energy partition theorem for quantum systems because: (i) it is universal; (ii) it is an extension of the formula for classical systems; (iii) it reduces to the energy equipartition theorem for high temperatures.

Acknowledgements The author would like to thank P. Hänggi and G.-L. Ingold for insightful discussions on various aspects of this work and P. Talkner for suggestions regarding the proof of normalization. The work supported by the Grant No. NCN 2015/19/B/ST2/02856.

Open Access This article is licensed under a Creative Commons Attribution 4.0 International License, which permits use, sharing, adaptation, distribution and reproduction in any medium or format, as long as you give appropriate credit to the original author(s) and the source, provide a link to the Creative Commons licence, and indicate if changes were made. The images or other third party material in this article are included in the article's Creative Commons licence, unless indicated otherwise in a credit line to the material. If material is not included in the article's Creative Commons licence and your intended use is not permitted by statutory regulation or exceeds the permitted use, you will need to obtain permission directly from the copyright holder. To view a copy of this licence, visit http://creativecommons.org/licenses/by/4.0/.

\section{References}

1. Huang, K.: Statistical Mechanics. Wiley, New York (1987)

2. Terletskií, Y.P.: Statistical Physics. North-Holland, Amsterdam (1971)

3. Waterston, J.J.: Note on the physical constitution of gaseous fluids and a theory of heat. R. Soc. Lond. 5, 604 (1846)

4. Boltzmann, L.: Über die Natur der Gasmoleküle. Wiener Berichte 74, 553 (1876)

5. Spiechowicz, J., Bialas, P., Łuczka, J.: Quantum partition of energy for a free Brownian particle: impact of dissipation. Phys. Rev. A 98, 052107 (2018)

6. Bialas, P., Spiechowicz, J., Łuczka, J.: Partition of energy for a dissipative quantum oscillator. Sci. Rep. 8, 16080 (2018)

7. Bialas, P., Spiechowicz, J., Łuczka, J.: Quantum analogue of energy equipartition theorem. J. Phys. A 52, 15LT01 (2019)

8. Feynman, R.P.: Statistical Mechanics. Westview Press, Reading, PA (1972)

9. Zwanzig, R.: Nonlinear generalized Langevin equation. J. Stat. Phys. 9, 215-220 (1973)

10. Caldeira, A.O., Leggett, A.J.: Quantum tunnelling in a dissipative system. Ann. Phys. (N.Y.) 149, 374-456 (1983)

11. Hänggi, P., Ingold, G.L.: Fundamental aspects of quantum Brownian motion. Chaos 15, 026105 (2005)

12. Callen, H.B., Welton, T.A.: Irreversibility and generalized noise. Phys. Rev. 83, 34-40 (1951)

13. Kubo, R.: The fluctuation-dissipation theorem. Rep. Prog. Phys. 29, 255-284 (1966)

14. Landau, L.D., Lifshitz, E.M.: Statistical Physics, Part I. Pergamon, Oxford (1980)

15. Zubarev, D.N.: Nonequilibrium Statistical Thermodynamics. Consultants Bureau, New York (1974)

16. Kubo, R.: Statistical-mechanical theory of irreversible processes. J. Phys. Soc. Jpn. 12, 570-586 (1957)

17. Martin, P.C., Schwinger, J.: Theory of many-particle systems. Phys. Rev. 115, 1342-1373 (1959)

18. Ford, G.W.: The fluctuation-dissipation theorem. Contemp. Phys. 58, 244 (2017)

Publisher's Note Springer Nature remains neutral with regard to jurisdictional claims in published maps and institutional affiliations. 